\title{
Prof. Dr. habil. Peter Glodek 70 Jahre
}

Am 16. Dezember 2004 vollendet Univ. Prof. em. Dr. PETER GLODEK sein 70. Lebensjahr. Persönlich und stellvertretend für viele Kollegen aus der Wissenschaft, Partnern aus der Zuchtpraxis, ehemaligen Mitarbeitern und Schülern nutze ich diese Gelegenheit unserem hochverehrten Jubilar sehr herzlich zu gratulieren. Sein Lebenswerk wurde in dieser Zeitschrift und an anderen Stellen bereits umfangreich gewürdigt, so dass hier Biographisches nur fragmentarisch erwähnt werden soll.

Im mecklenburgischen Bütow geboren, konnte er nach landwirtschaftlicher Praxisausbildung und Studium der Landwirtschaftswissenschaften in Berlin und Göttingen letzteres 1960 abschließen. Sein Lehrer FRITZ HARING lenkte die am Göttinger Institut beginnenden wissenschaftlichen Arbeiten Peter Glodek's auf das Gebiet der damals hochaktuellen Züchtung eines deutschen Fleischschweines, was 1962 zur Verteidigung seiner Dissertationsschrift mit dieser Thematik führte. Die Göttinger Schule sollte die Weichen für seine spätere wissenschaftliche Tätigkeit in Richtung Populationsgenetik und Züchtung mit vorrangigem Anwendungsbezug zum Schwein stellen. Diesem Ziel dienten auch seine später folgenden umfangreichen Studienaufenthalte in den damals weltweit besten Schulen der Tierzuchtwissenschaft bei FALCONER und ROBERTSON in Edinburgh sowie COMSTOCK und REMPEL in Minnesota. Zurück in Göttingen widmete er sich zunächst den Arbeiten zur Entwicklung von Selektionsindizes beim Schwein unter Einbeziehung mehrerer Merkmale und Informationsquellen insbesondere für die Eberselektion. Er habilitierte sich 1970 mit dieser Thematik. Im Jahr 1971 übernahm er als wissenschaftlicher Rat in der Nachfolge von RUTH GRUHN die Professur für Haustiergenetik und wurde 1973 nach seiner Berufung als ordentlicher Professor, Ordinarius für Tierzucht und Haustiergenetik am Göttinger Institut. Es folgten fast 30 Jahre eines erfolgreichen Wirkens in Lehre und Forschung an dieser ehrwürdigen und traditionsreichen Bildungsstätte. Aus seinem Selbstverständnis heraus, galt sein ganzes Augenmerk der Förderung des wissenschaftlichen Nachwuchses, den er bewusst in seine Forschungsprojekte einband. Zahlreiche seiner vielen ehemaligen Diplomanden, Doktoranden und Habilitanden, die ihm bis heute dankbar verbunden sind, werden sich sicher den heutigen Glückwünschen anschließen.

Zahlreich und vielfältig sind die Arbeitsfelder seiner wissenschaftlichen Tätigkeit, die in dieser Laudatio leider nicht umfassend dargestellt werden können. Genannt seien aus dem breiten Spektrum, neben Untersuchungen beim Rind und Pferd, vor allem beim Schwein Arbeiten zu Verbesserungen der Leistungsprüfungen und Zuchtwertschätzung, der züchterischen Mög- 
lichkeiten zur Verbesserung der Wirtschaftlichkeit der Schweineerzeugung, der Fleischbeschaffenheit und Stressempfindlichkeit oder zuchtmethodische Forschungsprojekte. Bei allen diesen, oft der Zeit vorausschauenden, Arbeiten war es stets sein Bestreben die Ergebnisse der Forschung unmittelbar in die praktische Nutzanwendung zu überführen. Mit Tatkraft und Engagement in der Organisation und in Zusammenarbeit mit den Tierzuchtorganisationen fanden sich die Ergebnisse in der Zuchtpraxis wieder und wirkten beispielhaft auch über die Landesgrenze hinaus. Er war es auch mit dessen Hilfe, in der Rückbesinnung auf die Nutzung von Hybrideffekten beim Schwein in Deutschland, das Bundeshybridzuchtprogramm entwickelt wurde, welches nach Zahlen des ZDS noch heute zu den führenden Schweinezuchtprogrammen in Europa zählt. Neben solchen Projekten, deren Nutzen die deutschen Schweineproduzenten realisiert haben, kennen wir die Ergebnisse seiner Arbeit aus einer sehr großen Anzahl von Veröffentlichungen und Vorträgen, Büchern und Buchbeiträgen, die sein äußerst kreatives Schaffen nachhaltig belegen. Hält man nach gemeinsamen Prinzipien für alle diese von PETER GLODEK durchgeführten, angeregten oder wissenschaftlich betreuten Arbeiten Ausschau, so bieten sich die Schlüsselworte: Aktualität, Pionierfunktion, methodische Innovation, wissenschaftliche Aussagefähigkeit und praktische Relevanz an. Als Hochschullehrer wirkte er auf dem Gebiet der Tierzucht und Populationsgenetik außerordentlich erfolgreich. Er verstand es sein umfangreiches fachliches Wissen in sehr anschaulicher Weise zu vermitteln. Seine brillante Rhetorik machten seine Vorlesungen für die Studenten stets zu einem Erlebnis. Das galt übrigens auch für seine Vorträge, die, unabhängig ob bei wissenschaftlichen Tagungen im In- und Ausland oder Züchterforen, sein Ansehen in Wissenschaft und Praxis gleichermaßen gefördert haben. Man musste nicht nachdenken was wohl oder wie es gemeint war, sondern es genügte hinzuhören, was er sagt.

Nach der Wiedervereinigung war sein bereitwillig gegebener Rat bei der Neuorientierung der Zuchtarbeit in den ostdeutschen Bundesländern gefragt und stets war und ist er gern gesehener Gast auf Züchtertagungen in diesen Zuchtgebieten. Nicht unerwähnt bleiben darf sein Engagement in der Schweinezucht einiger osteuropäischer Länder.

Erfolgreiche Arbeit erzeugt Nachfrage und so hat PETER GLODEK zahlreiche ehrenamtliche Tätigkeiten wahrgenommen. Erwähnt seien nur sein Wirken als geschäftsführender Direktor des Tierzuchtinstitutes, als Dekan, sein Engagement in mehreren Wahlämtern der Deutschen Gesellschaft für Züchtungskunde, die ihm im Jahre 2000 für seine Verdienste die „Hermannvon-Nathusius-Medaille“ verlieh, seine Mitarbeit im Redaktionsausschuss der „Züchtungskunde“, in der Europäischen Vereinigung für Tierproduktion, der deutschen Forschungsgemeinschaft, in wissenschaftlichen Beiräten, so bis heute in dem des Forschungsinstitutes für die Biologie landwirtschaftlicher Nutztiere in Dummerstorf.

Unmittelbar nach der Wiedervereinigung war er es, der mich als Schriftleiter des „Archiv für Tierzucht“ bereitwillig, uneigennützig, hilfsbereit und sachkundig sowie durch wertvolle Anregungen tatkräftig unterstützte und bis heute im Redaktionskollegium mitwirkt. Nicht zuletzt hat durch seine verdienstvolle Mitarbeit unsere Zeitschrift ihr heutiges internationales Niveau erreicht, wofür ihm mein ganz besonderer, persönlicher Dank gilt. Gern erinnere ich mich an die zahlreichen freundschaftlichen Treffen mit ihm. Damit verbinden sich für mich seine fachliche Kompetenz, kollegiale Zusammenarbeit, sein immer gern gegebener Rat oder seine helfende Kritik und mein Wunsch ist es auch in der kommenden Zeit noch auf seine Unterstützung zählen und viele herzliche Begegnungen mit ihm erleben zu dürfen.

Wir, Kollegen Tierzüchter, haben allen Anlass uns bei PETER GLODEK, einem Großen der deutschen Tierzucht, zu bedanken. Für die Zukunft wünsche ich dem Jubilar alles erdenklich Gute, Gesundheit, viel Freude bei allen seinen Unternehmungen, vor allem aber noch viele schöne gemeinsame Jahre mit seiner Gattin, seiner Familie und Freunden in seinem tätigen Ruhestand. 